To appear in Vehicle System Dynamics

Vol. 00, No. 00, Month 20XX, 1-

\title{
Circuit Racing, Track Texture, Temperature and Rubber Friction
}

\author{
R. S. Sharp ${ }^{a *}$ and P. Gruber ${ }^{a}$ and E. Fina ${ }^{a}$ \\ ${ }^{a}$ Department of Mechanical Engineering Sciences, University of Surrey, Guildford GU2 7XH, \\ $U K$
}

(November 2015)

\begin{abstract}
Some general observations relating to tyre shear forces and road surfaces are followed by more specific considerations from circuit racing. The discussion then focuses on the mechanics of rubber friction. The classical experiments of Grosch are outlined and the interpretations that can be put on them are discussed. The interpretations involve rubber viscoelasticity, so that the vibration properties of rubber need to be considered. Adhesion and deformation mechanisms for energy dissipation at the interface between rubber and road and in the rubber itself are highlighted. The enquiry is concentrated on energy loss by deformation or hysteresis subsequently. Persson's deformation theory is outlined and the material properties necessary to apply the theory to Grosch's experiments are discussed. Predictions of the friction coefficient relating to one particular rubber compound and a rough surface are made using the theory and these are compared with the appropriate results from Grosch. Predictions from Persson's theory of the influence of nominal contact pressure on the friction coefficient are also examined. The extent of the agreement between theory and experiment is discussed. It is concluded that there is value in the theory but that it is far from complete. There is considerable scope for further research on the mechanics of rubber friction.
\end{abstract}

Keywords: rubber, friction, deformation, hysteresis

\section{Introduction}

Some general observations relating to tyres, road surfaces and rubber friction are followed by comments more specifically related to circuit racing. The focus then is on the mechanics of rubber friction, especially that part of the friction which is thought to be associated with deformation of the rubber as it interacts with irregularities in the rigid surface over which it is travelling. In particular, Grosch's classical experiments [1] are described and the interpretations of the results obtained are discussed. Then, Persson's hysteresis-loss theory [2] is outlined and used to reproduce some of Grosch's results to test the capability of the theory. Conclusions are drawn at the end.

\section{Observations}

Road vehicle manoeuvring depends almost entirely on tyre shear forces. It is indisputable that such forces are vital in vehicle dynamics and control. The shear forces depend on friction between rubber and road and they depend on the chemical nature of the road

\footnotetext{
${ }^{*}$ Corresponding author. Email: robinsharp37@talktalk.net
} 
surface (e.g., asphalt or concrete) and its texture, the rubber compound, rubber and road surface temperatures and the sliding speed of the rubber relative to the road.

Race track surfaces differ from each other. In fact some track segments are significantly different in texture and possibly chemical composition from others. In racing, the maximum possible forces are required. New tracks, so-called "green" tracks, change their nature with usage as rubber is deposited on the surface near to the racing line. It is often reported that the maximum shear forces increase as a track "rubbers-in" for tyres with similar compounding to those tyres doing the previous running but that different tyres do not do so well. If rain falls on a rubbered-in track, the racing line changes as the friction levels on the presumed-smoother part of the track decrease, while the green track is relatively unaffected.

The very-high friction tyres used in motor racing are especially compounded and have relatively high wear rates and short lives. They are very sensitive to track and tyre temperatures and thus need to be run "on-tune" to give good performance.

\section{Grosch's experiments}

Grosch's experiments involved four very different rubber compounds, isomerised natural rubber (INR), acrilonitrile-butadiene rubber (ABR), styrene-butadiene rubber (SBR), and butyl rubber (Butyl), sliding across mainly two surfaces, a smooth glass, described as wavy glass, and rough silicon carbide in the form of emery cloth. Surface profile details were not specified but the emery cloth was 180 -grade. The glass or silicon carbide surfaces were slid under a nominally flat rubber specimen, loaded by hanging weight against the surface. In some of the rough-surface experiments, the surface was dusted with a thin layer of talcum powder to interfere with the surface to surface interactions to some extent. Very low sliding velocities were employed to avoid significant heating at the rubber to surface contact.

The whole active part of the experiment was enclosed in a temperature-controlled enclosure and sliding speed and friction force measurements were made for temperatures distributed between $-50^{\circ} \mathrm{C}$ and $100^{\circ} \mathrm{C}$. For a given sliding speed, the shear force is indicative of the energy dissipation at the rubber to surface interface. Those mechanisms that lead to energy dissipation are therefore of interest. Grosch's experiments are illustrated by Figure 1 and his apparatus is shown in Figure 2.

\section{Experimental results}

Some of Grosch's measured friction coefficients as functions of sliding speed and temperature are shown in Figure 3 for INR sliding on silicon carbide and for ABR sliding on wavy glass.

Such raw results can be transformed to master-curve form using the frequencytemperature equivalence established in [3] for a range of polymers, through the plotting of $a_{T} V$ instead of $V$, where $a_{T}$ is given by:

$$
\log _{10}\left[a_{T}\right]=\frac{-8.86\left(T-T_{s}\right)}{101.5+T-T_{s}}
$$

Here, $T_{s}$ is a standard temperature for each rubber, approximately $50^{\circ} \mathrm{C}$ above the respective glass transition temperature and $T$ is the test temperature. The transformation 


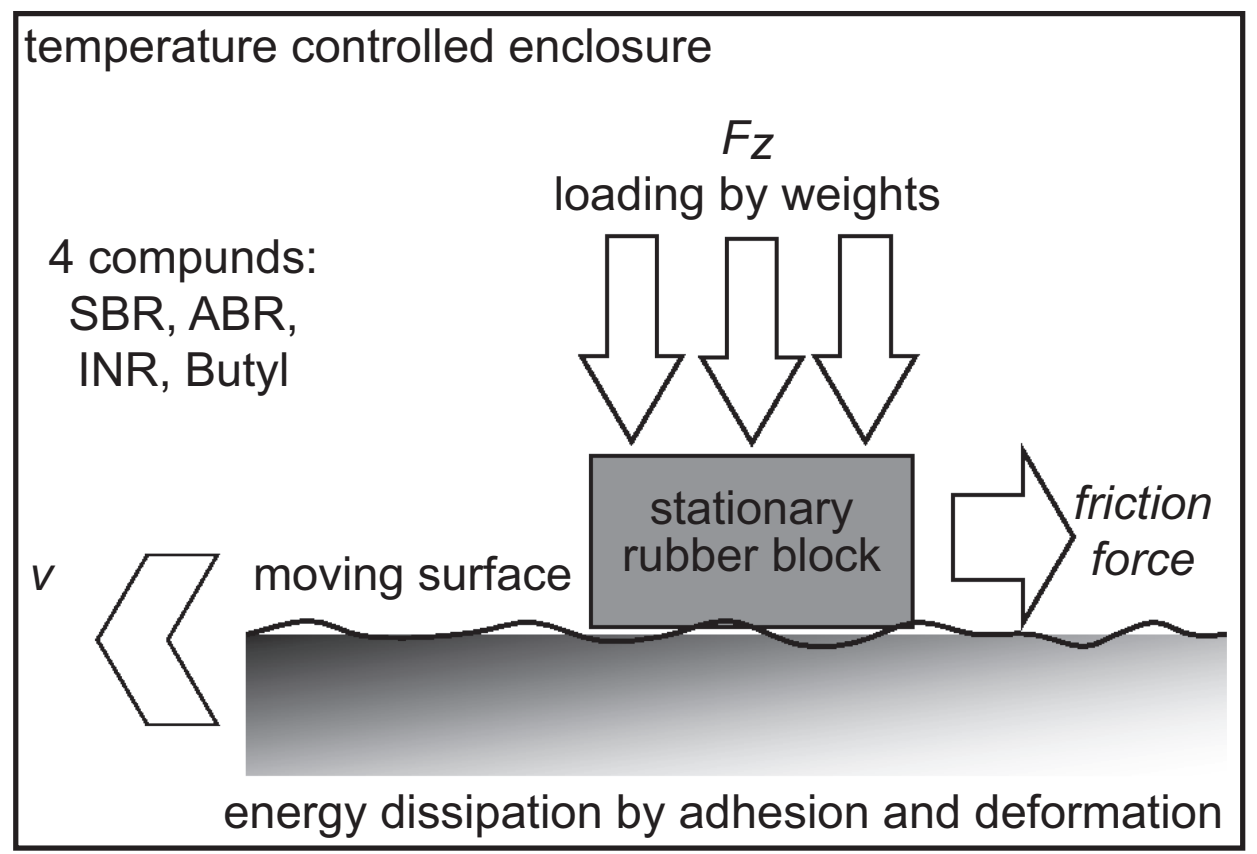

Figure 1. Illustration of the sliding surface and the stationary rubber specimen in the temperature-regulated enclosure of Grosch's experiments.

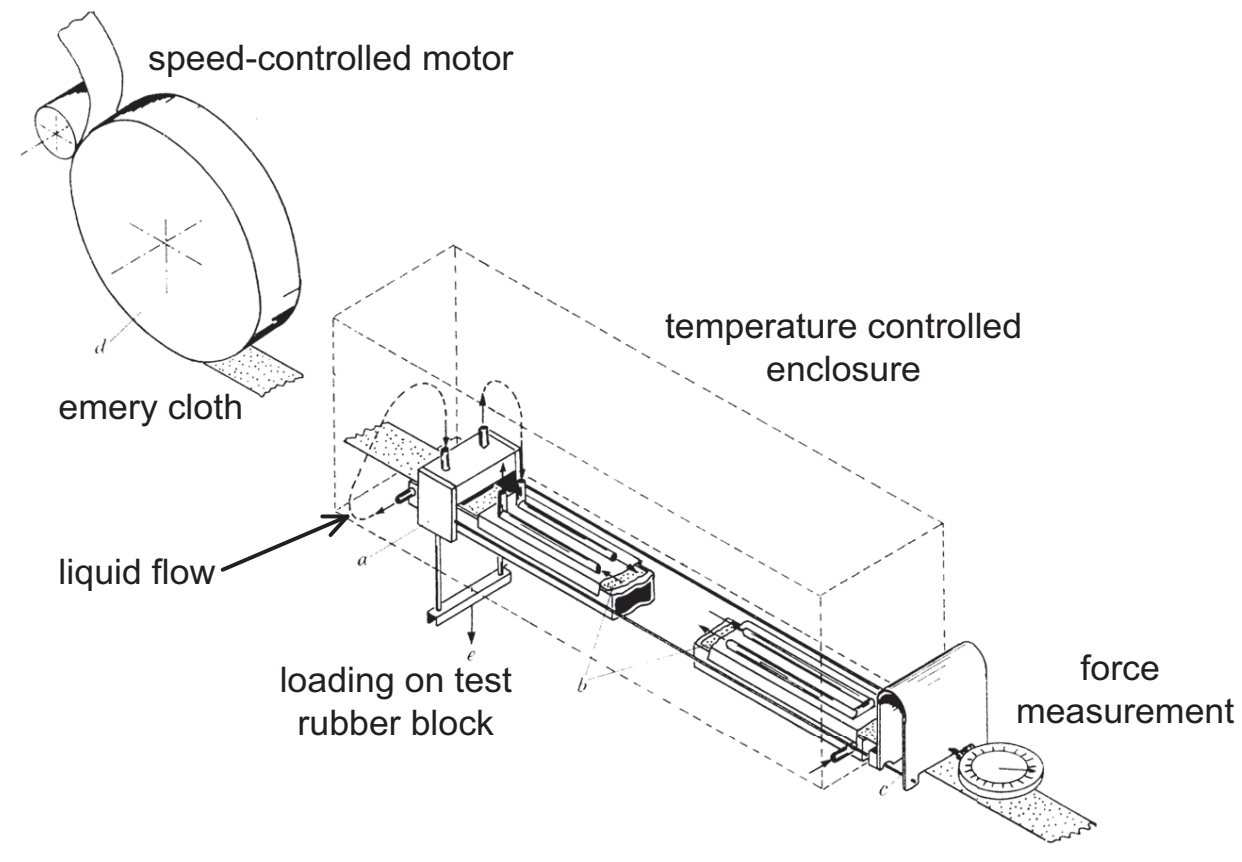

Figure 2. Schematic representation of Grosch's friction measurement apparatus, adapted from [1].

allows all the results to be referred to some arbitrarily chosen temperature. Example master curves are shown in Figures 4, 5, 6 and 7.

The fact that this WLF transformation reduces all the raw results to master curves shows that the friction arises from viscoelasticity and that the low sliding speeds employed were sufficient to prevent significant heating of the rubber. Some of the master curves show two distinct friction peaks and Grosch associated the one occurring at a lower 

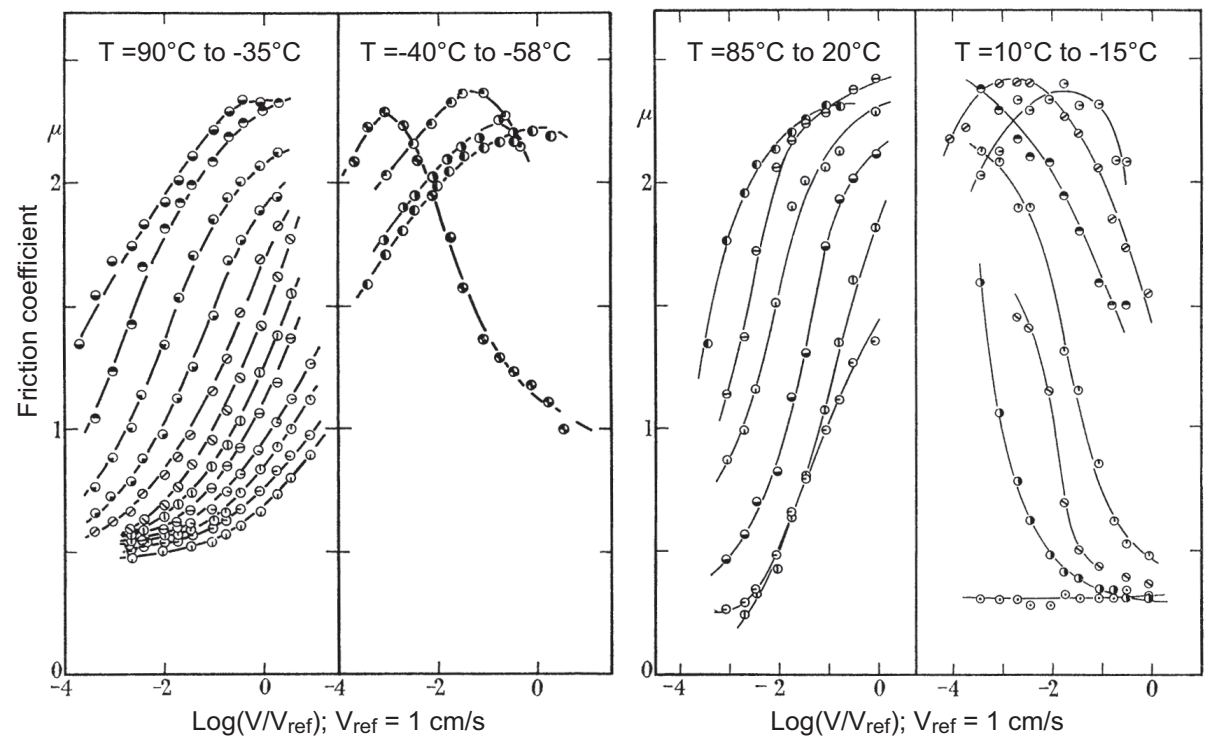

Figure 3. Friction coefficient as a function of sliding speed at different test temperatures for INR sliding on silicon carbide cloth (left) and for ABR sliding on wavy glass (right), adapted from [1].
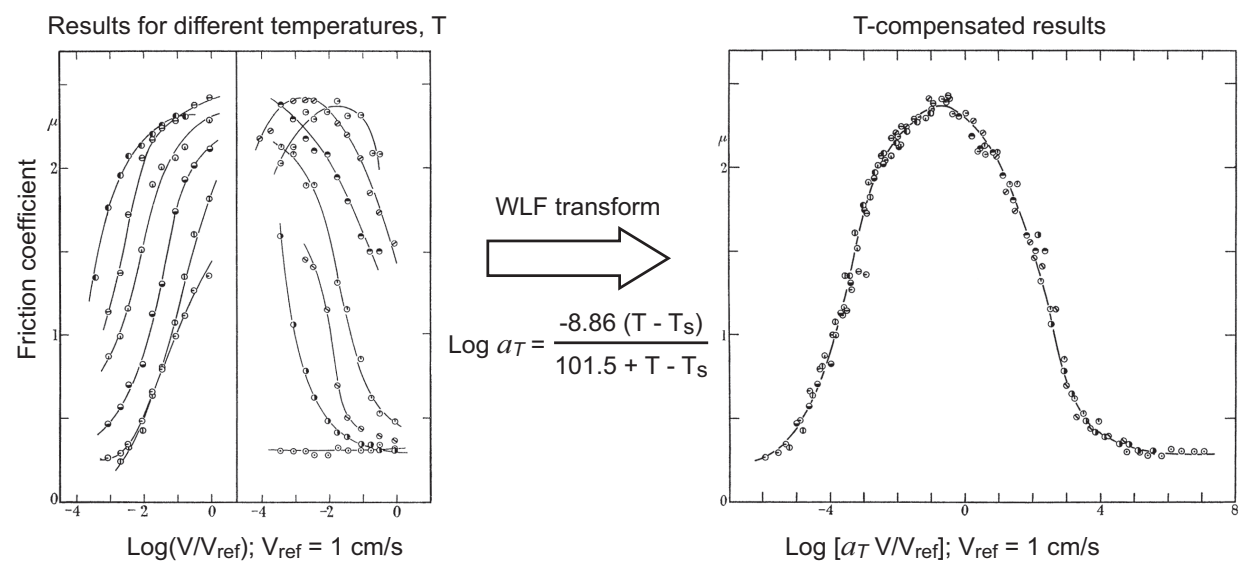

Figure 4. Temperature-compensation of the raw friction results by the WLF transform gives a master curve for ABR on wavy glass, adapted from [1].

sliding velocity with adhesion and the other with deformation. In keeping with this interpretation, dusting the surface with talcum powder interfered with the lower peak friction much more than with the higher one. Grosch also found that the ratio of the sliding velocity giving peak adhesion friction, $V_{S P}$, to the frequency where the rubber has its loss modulus peak, $\omega_{L M P}$, is the same for all four rubbers, with $V_{S P} \approx 6 E-$ $9 \omega_{L M P} /(2 \pi)$. Further, the ratio of the sliding velocity giving peak deformation friction, $V_{R P}$, to the frequency where the rubber has its loss tangent peak, $\omega_{L T P}$, is the same for all four rubbers, with $V_{R P} \approx 1.5 E-4 \omega_{L T P} /(2 \pi)$, see Section 5. Correspondingly, the ratio of $V_{S P}$ on a smooth surface to $V_{R P}$ on a rough surface is given by $6 E-9 \omega_{L M P} / 1.5 E-4 \omega_{L T P}$ which implies that, if $\omega_{L M P}$ and $\omega_{L T P}\left(\omega_{L M P}>\omega_{L T P}\right)$ for a particular material are far apart, then the friction peaks for that material will be close together and conversely. The factors $6 E-9$ and $1.5 E-4$ appearing in the above relationships are dimensions in metres; the former being molecular and consistent with regular bond formation, stretching and rupture, giving rise to excitation of the rubber at its most dissipative frequency. The 


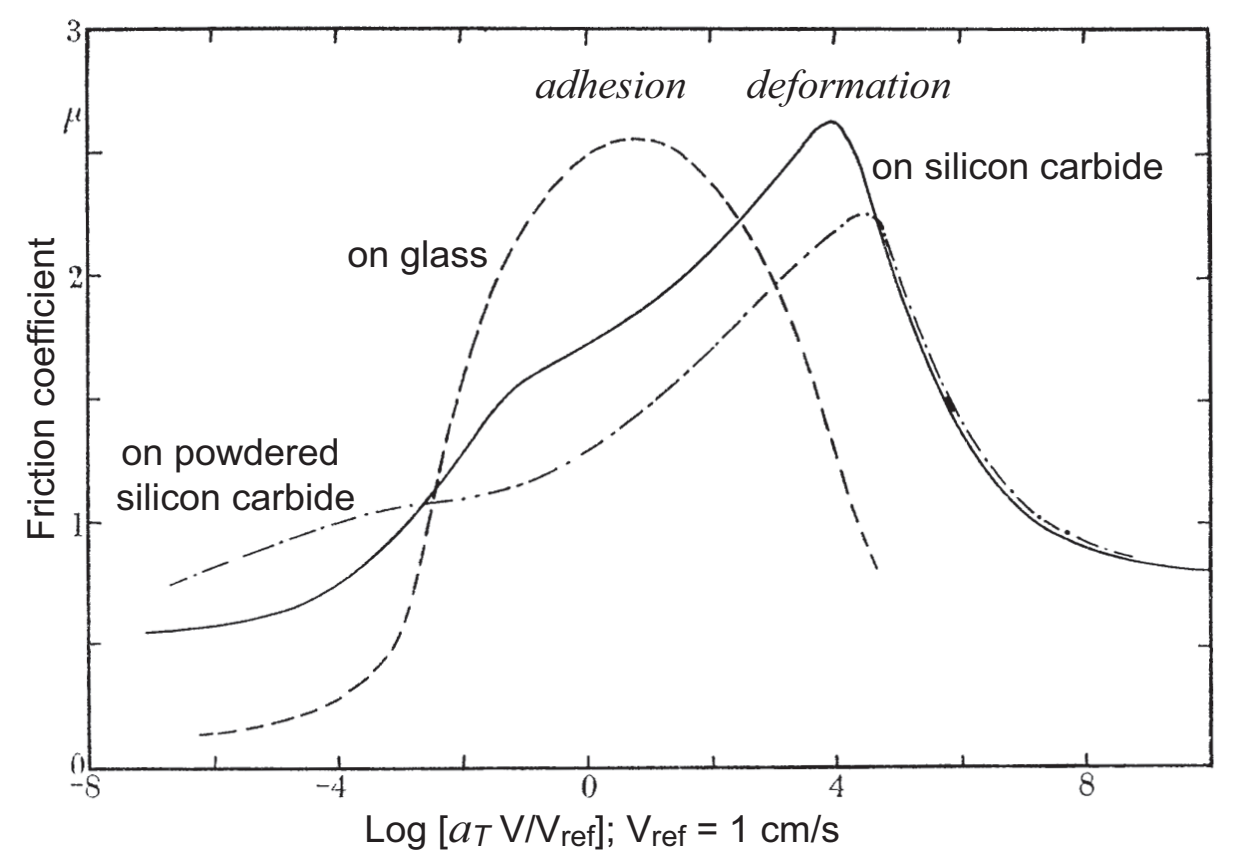

Figure 5. Grosch master curves for SBR at $20^{\circ} \mathrm{C}$ on glass and silicon carbide surfaces, adapted from [1].

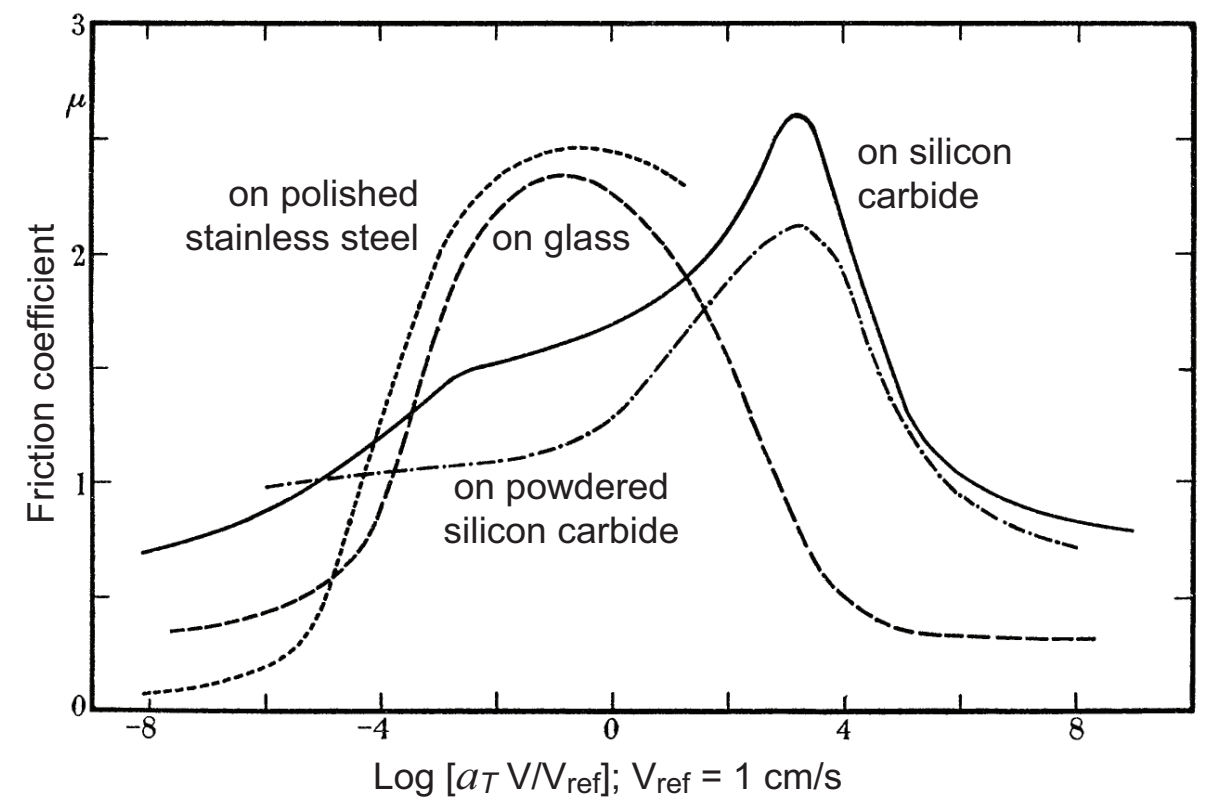

Figure 6. Grosch master curves for $\mathrm{ABR}$ at $20^{\circ} \mathrm{C}$ on glass and silicon carbide surfaces, adapted from [1].

latter is close to giving excitation where the rubber deformation and its consequent energy loss are maximal due to the irregularity of the surface over which it slides. Grosch noted that the average spacing of the abrasive particles in his emery cloth was $1.4 E-4 \mathrm{~m}$ and, thus, close to the $1.5 E-4 \mathrm{~m}$.

Grosch stated that the nominal pressure on the rubber up to 5.5E4 Pa, (that is, the load divided by the area of the rubber block projected onto the substrate), had negligible influence on the friction coefficient. In reviewing the influence of the loading in $[4,5]$, 


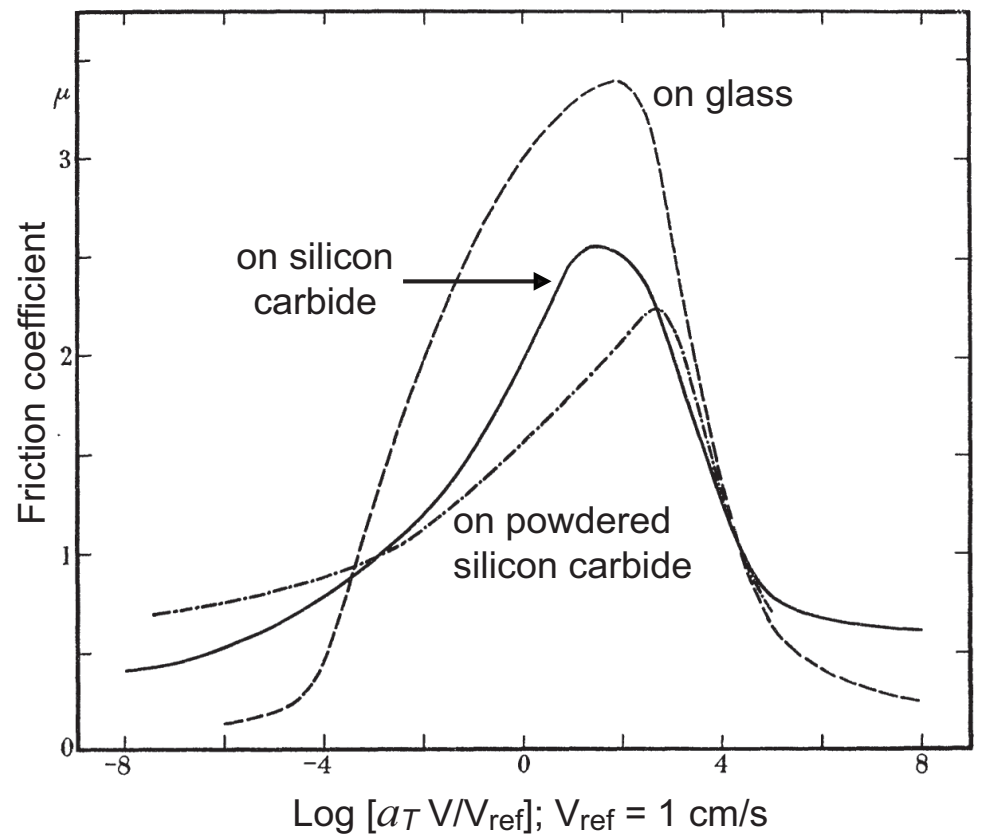

Figure 7. Grosch master curves for Butyl at $20^{\circ} \mathrm{C}$ on glass and silicon carbide surfaces, adapted from [1].

he said that for soft rubbers on smooth surfaces, the friction coefficient decreases with increasing load. For harder tyre-tread compounds on tracks of different asperity shapes and coarseness, the friction coefficient varies with loading to a power that ranges from -0.1 to +0.1 and the variation is often so small as to be negligible. Simple theory, using the idea that the friction will be proportional to the real area of contact, suggests that the friction coefficient is proportional to the nominal pressure to the power $-\left(\frac{1}{3}\right)[6]$ and some results for higher pressures conform to that pattern. Grosch, Schallamach and Persson have commented on the possibility that temperature influences might be mistaken for loading ones [5-7]. Some recent measurements [8] for a contemporary racing-tyre compound show the friction coefficient decreasing significantly with increasing nominal pressure in the range $1 E 5$ to $7 E 5 P a$, see Figure 8.

\section{Rubber vibration properties}

To understand the general shapes of the various master curves, the viscoelastic nature of rubber must be appreciated. The vibration properties are typically measured with a mechanical analyser, Figure 9, see

http://en.wikipedia.org/wiki/Dynamic_mechanical_analysis. The rubber specimen is contained within a temperature-controlled enclosure and strained at a controllable frequency and amplitude. Stress and strain are measured and the stress is found to be phase-shifted relative to the strain, indicating energy storage and energy dissipation, both varying markedly with frequency.

Typical results for testing at constant temperature are illustrated in Figure 10. When the forcing frequency is low, the material is compliant and not very dissipative. At the other extreme, when the forcing frequency is very high, the material becomes very stiff, typically three orders of magnitude stiffer than at low frequencies. In the transition region, the energy dissipation increases to a peak at a frequency called $\omega_{L M P}$ and falls 


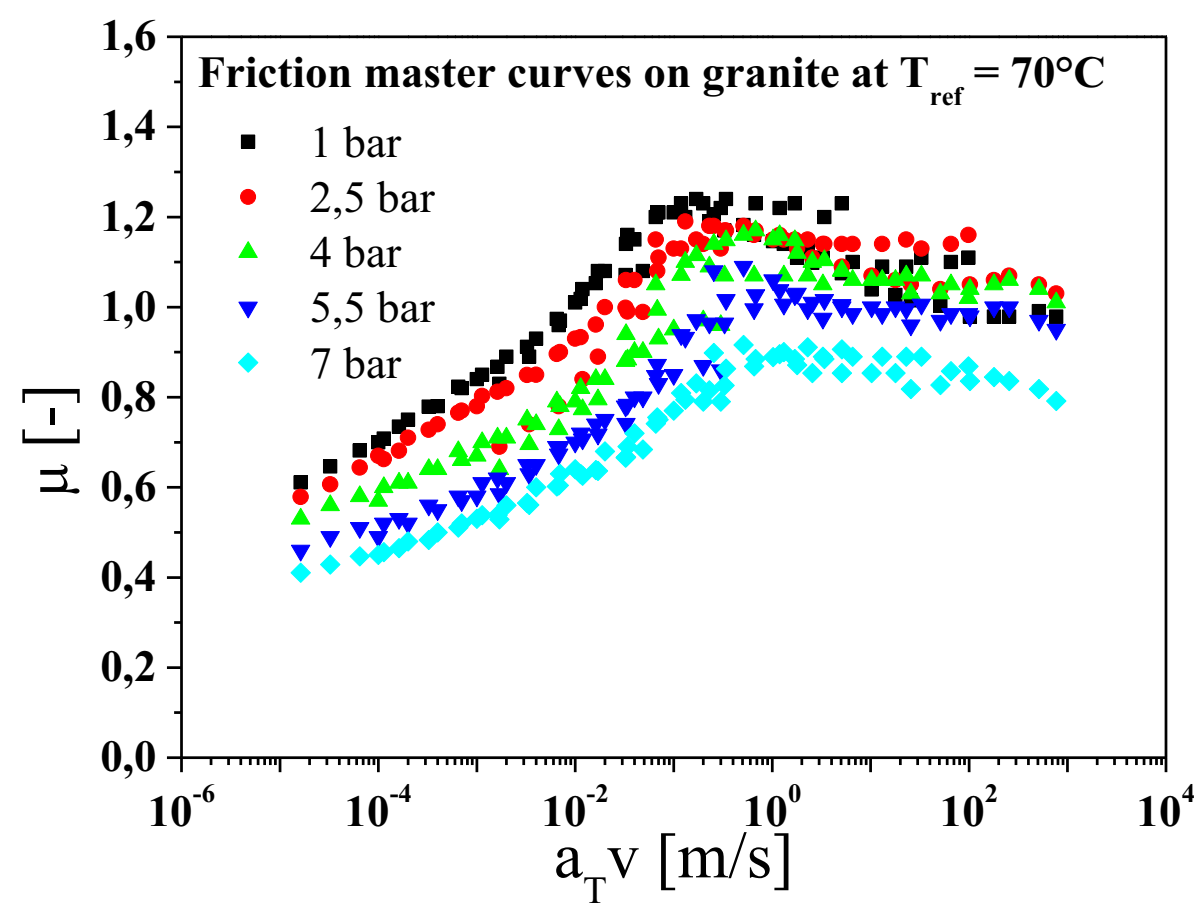

Figure 8. Friction coefficients as a function of normalised sliding speed and nominal contact pressure for a racingtyre compound on a coarse granite surface, from [8].

again afterwards. The ratio of the loss modulus to the storage modulus is also significant, constituting the loss tangent. The frequency at which the loss tangent peaks is called $\omega_{L T P}$.

Such results vary with the amplitude of the vibrations used in the testing. The greater the amplitude is, the more compliant the rubber appears to be, see Figure 11.

\section{Theory of rubber friction}

A theory for adhesion was developed by Schallamach [1] and subscribed to by Grosch [1] and by Persson [1]. Chemical bonds between rubber and substrate are formed and broken with regularity. The rate at which bonds are formed is governed by an activation energy that is lowered by frictional stress and by temperature reduction. Conversely the rate is decreased by increasing sliding speed, since the time available for bond formation is then lessened. Maximum adhesion can be expected when the two processes balance. This theory has not yet been developed into a quantitative state with experimental confirmation.

In contrast to this situation, Persson's hysteresis theory $[1,1,2,7]$ is quite complete. In this context, the interaction between the rubber and the surface can be considered most simply by first thinking of the surface as having a sinusoidal profile and later as having many wavelength constituents, Figure 12 . The compliant rubber conforms to the rigid surface to a degree that depends on its normal loading and its stiffness. Initially, each wave can be considered individually, as if only one wavelength were present but, in the more complex and general case, the conformity of the rubber to the short wave irregularities depends on the presence of long waves because these affect the normal loading between the surfaces. 

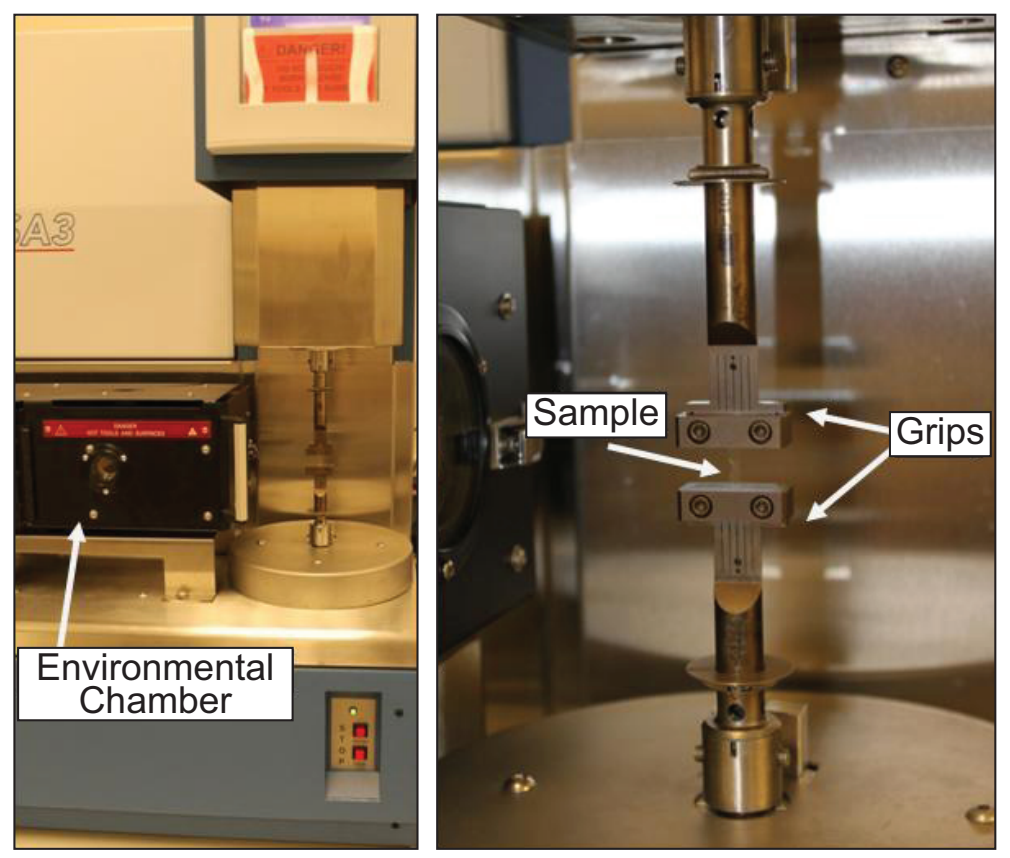

Figure 9. A commercial mechanical analyser for the measurement of the dynamic properties of rubber. On the right is a close-up of the test specimen.

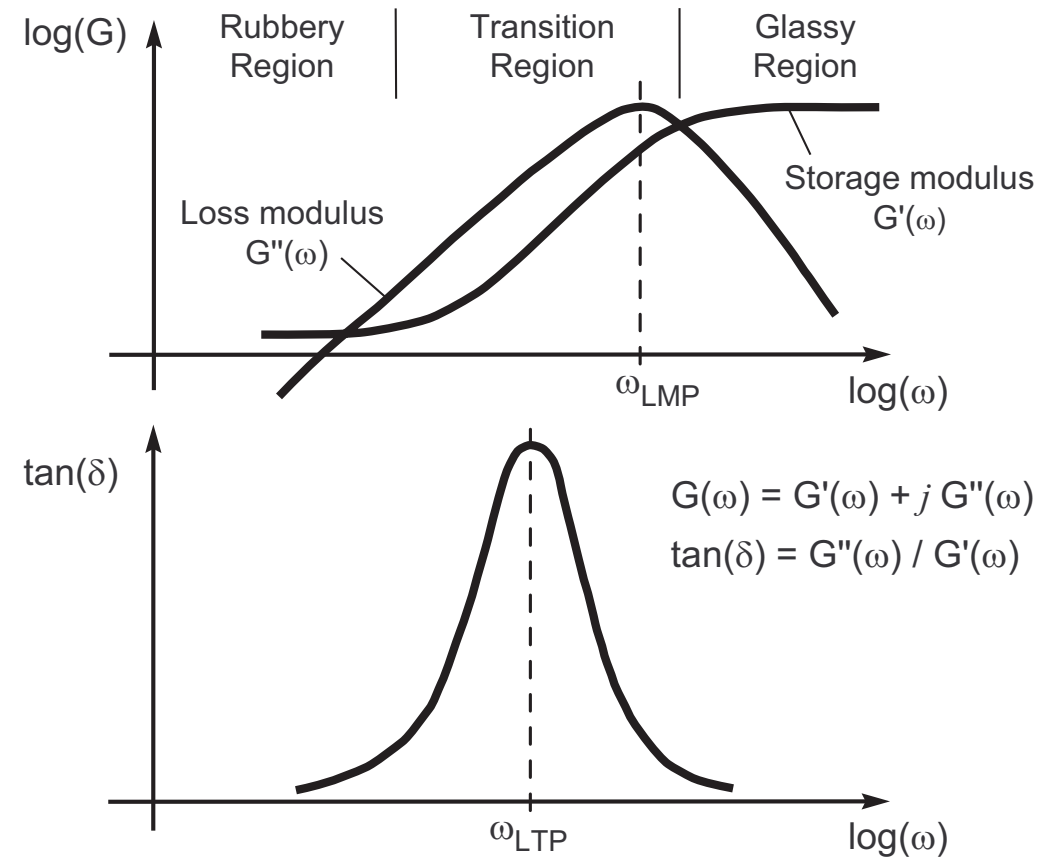

Figure 10. Typical shear vibration response properties of rubber.

In the simple case in which the surface is sinusoidal, it can be expected that the energy dissipation will be maximal when the sliding velocity is such that the deformation frequency is $\omega_{L M P}$. On this basis, a friction peak at a particular sliding velocity is anticipated. Persson's deformation theory [2] gives: 

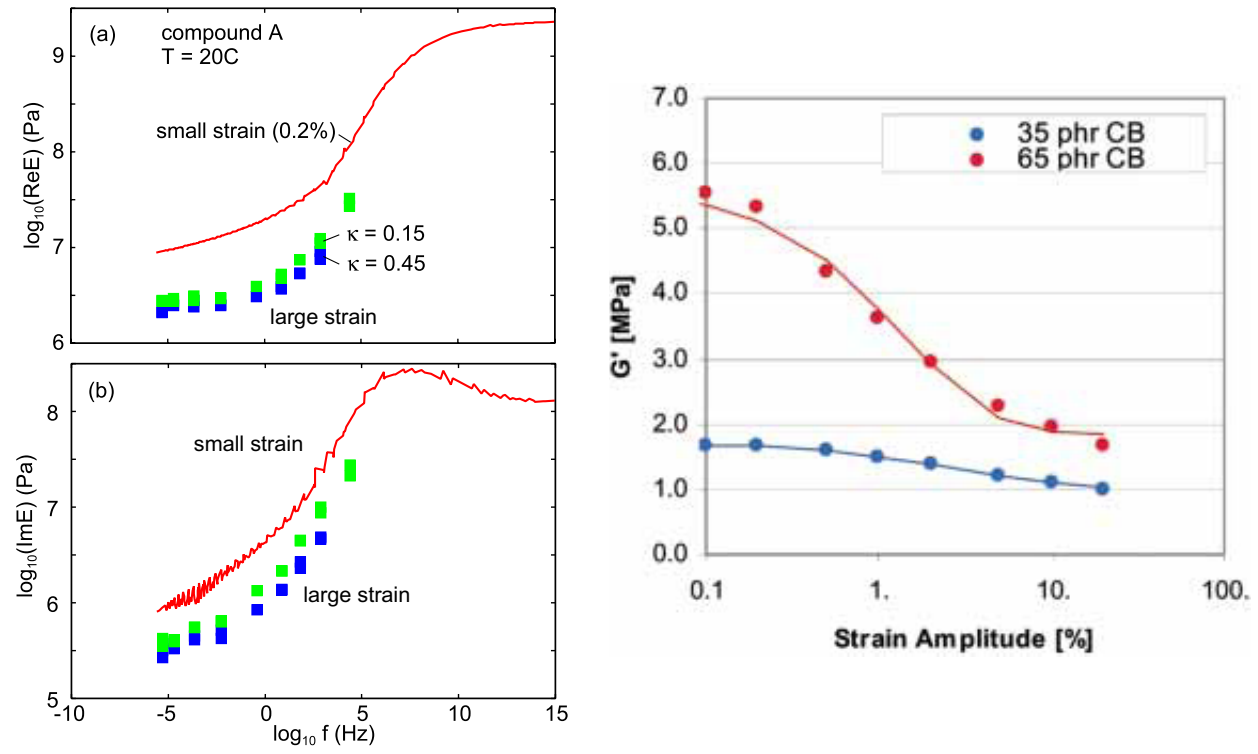

Figure 11. Amplitude-dependence of vibration response properties of rubber as given (left) in [9] and (right) in $[1]$.

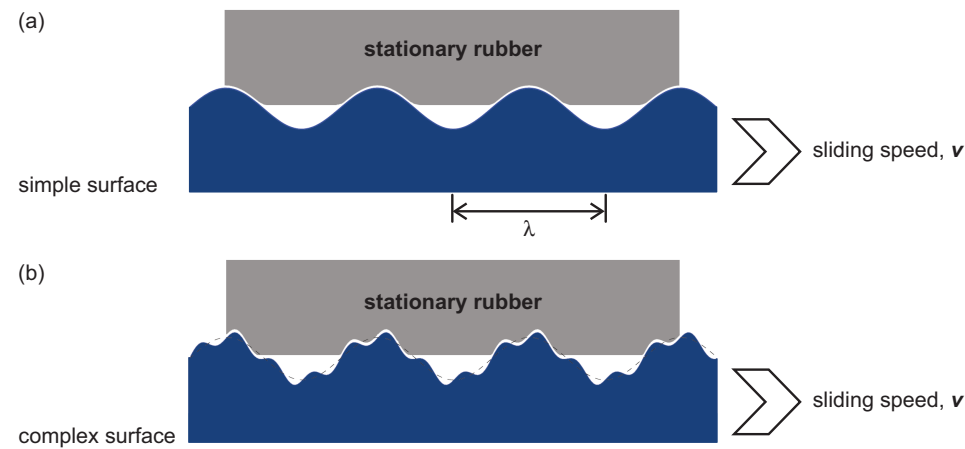

Figure 12. Schematic representation of the interaction between the rubber block and (a) a generic wave undulation of length $\lambda$ and (b) a more complex wave with many wavenumber components; the proportionality between the deformation frequency and the sliding speed should be clear.

$$
\mu=\frac{1}{2} \int_{q_{0}}^{q_{1}} q^{3} C(q) P(q) \mathrm{d} q \int_{0}^{2 \pi} \cos \phi \operatorname{Im}\left(\frac{2 G(q V \cos \phi, T)}{\sigma_{z}(1-\nu)}\right) \mathrm{d} \phi
$$

where $G(\omega)$ is the complex shear modulus of the rubber compound, $C(q)$ the surface power spectrum, and $T$ is the compound's temperature. The frequency dependency of the complex modulus $G(\omega)$ is written as sliding velocity dependency since $\omega=q V \cos (\phi)$; where $\phi$ is the angle between the sliding direction and the orientations of the road undulations, described by the wavenumber $q$. The terms $\sigma_{z}$ and $\nu$ are the nominal pressure applied to the rubber block and the Poisson's ratio of the rubber compound respectively, the latter assumed to have the constant value $0.5 . G(\omega)$ is related to the tensile modulus $E(\omega)$ by $E(\omega)=2(1+\nu) G(\omega)$.

However, under the nominal vertical stress $\sigma_{z}$, the surface asperities do not fully penetrate the rubber and only a partial contact between the surface and the rubber block can 
be achieved. Therefore, the power spectrum cannot contribute in full to the hysteresis friction. This aspect is taken into account in Equation (1) through the factor $P(q)$, which is the ratio of the real contact area to the nominal contact area, the latter corresponding to complete filling of the surface voids by the rubber.

$$
P(q)=\frac{2}{\pi} \int_{0}^{\infty} \frac{\sin (x)}{x} \exp \left[-x^{2} F(q)\right] d x
$$

with $\mathrm{F}(\mathrm{q})$ given by:

$$
F(q)=\frac{1}{8} \int_{q_{0}}^{q_{1}} q^{3} C(q) \int_{0}^{2 \pi}\left|\frac{2(1+\nu) G(q V \cos (\phi), T)}{\left(1-\nu^{2}\right) \sigma_{z}}\right|^{2}
$$

It can be shown that, in the usual case when the nominal stress $\sigma_{z}$ is $\ll G(0)$, the compound static shear stiffness, the $P(q)$ factor can be approximated by, see [2]:

$$
P(q)=\left[\frac{\pi}{8} \int_{q_{0}}^{q_{1}} q^{3} C(q) \mathrm{d} q \int_{0}^{2 \pi}\left|\frac{2 G(q V \cos (\phi), T)}{(1-\nu) \sigma_{z}}\right|^{2} \mathrm{~d} \phi\right]^{-1 / 2}
$$

Equation (4) shows that the normalized area of contact is directly proportional to the vertical pressure $\sigma_{z}$ and inversely proportional to the stiffness of the compound. In other words, the area of contact between the rubber block and the surface increases with increasing vertical load and/or reducing compound stiffness. The notation employed for Persson's hysteresis theory is summarised in Table 1.

Table 1. Notation used in Persson's hysteresis theory.

\begin{tabular}{|c|c|}
\hline Symbol & Meaning \\
\hline$\mu$ & coefficient of friction \\
$\mathrm{C}(\mathrm{q})$ & surface displacement spectral density function \\
$\mathrm{P}(\mathrm{q})$ & ratio of actual contact area to nominal contact area \\
$\mathrm{F}(\mathrm{q})$ & function needed to evaluate $\mathrm{P}(\mathrm{q})$ precisely \\
$q_{0}, q_{1}$ & lower and upper wavenumbers included in integrals \\
$T_{q}$ & temperature \\
$\omega$ & vibration circular frequency \\
$E(\omega)$ & complex tensile modulus of rubber \\
$G(\omega)$ & complex shear modulus of rubber \\
$\mathrm{x}$ & contact-pressure-related variable in the stress probability \\
& distribution function [1, 2] \\
$\nu$ & Poisson's ratio for rubber (assumed constant at 0.5) \\
$\mathrm{V}$ & sliding velocity \\
$\sigma_{z}$ & nominal normal stress \\
\hline
\end{tabular}

Notwithstanding the marked amplitude-dependence of rubber elasticity, in Equation (1) the material is treated as linear when integrals over the $q_{0}$ to $q_{1}$ range are evaluated. The complex modulus $G(\omega)$ describes both stiffness and damping of the rubber compound. Stiffness and damping have opposite effects on the energy dissipated by the rubber and hence on the calculated friction. For a given sliding velocity and normal pressure, a stiffer compound will conform to surface undulations less and the volume of 
rubber distorted will be reduced. Conversely, if the material is more dissipative, a given distortion will imply greater energy loss.

Heating of the material at the sliding interface as a consequence of the energy dissipation is included in Persson's later theory $[1,1,7]$ but this is not an issue in the present context due to the very low sliding velocities used by Grosch.

Persson [1] expresses the view that the contribution to the friction from hysteresis is dominant when the real contact area is a small proportion of the nominal area, associating this behaviour with rough surfaces. Subsequently, friction due to adhesion receives relatively little attention, despite that many measurements on rough surfaces, including roads, show a strong friction peak attributable to adhesion $[1,1,1,9]$.

\section{Surface and rubber data necessary in Persson's hysteresis theory}

For the quantitative prediction of the hysteretic component of friction using Persson's theory, the rubber compound's complex shear modulus $G(q V \cos (\phi))$ and the surface vertical displacement power spectral density function $C(q)$ in the wavenumber range $q_{0}$ to $q_{1}$ are needed. Also, the temperature-frequency shift factor, $a_{T}$, needs to be known. The best data available relates to SBR, for which Grosch specified the standard temperature, $T_{s}$, and the loss modulus, but not the storage modulus. SBR is commonly used in tyre manufacture. Neither did Grosch specify the surface roughness of his 180-grade silicon carbide cloth but a similar material, commercially known as "safety-walk", has been measured with a laser profilometer (AMES laser texture scanner, model 9200) to give $C(q)$, Figure 13. Grosch gives only the average spacing between abrasive particles as $1.4 E-4 \mathrm{~m}$. For the measured surface the wavenumber $q_{0}$ was found to be $\approx 2.0 E-4 \mathrm{~m}$. The cut-off wavenumber $q_{1}$ is determined here by the resolution of the profilometer. It is $\approx 10^{5.344} \mathrm{~m}^{-1}$ and corresponds to a wavelength of $\approx 28.5 \mu \mathrm{m}$. The cut-off wavenumber is in reality dictated by the cleanliness of the surface and it is difficult to know it with any certainty $[1,1,2]$. Rubber wear particles occupy the interface between rubber and track, reducing the deformation of the rubber and hence the hysteretic friction. The rough surface of the emery cloth is naturally of greater interest than that of the glass in the context of hysteretic friction. For SBR, the required storage moduli are initially obtained from the literature [2, 2], Figure 14. The materials will be referred to subsequently as "Grosch-Klüppel" and as "Grosch-Fletcher". The frequencies $\omega_{L T P}$ at which the loss tangents peak agree well with Grosch's account and the two storage moduli are similar functions of frequency, although quite different in magnitude.

\section{Results}

Using the numerical data given in Section 7 for SBR sliding on silicon carbide cloth, friction coefficients as shown in Figure 15 are generated. As in Grosch's testing, the rubber state is varied in these computations by notionally employing low temperatures so that the sliding velocities can be kept low and the heating negligible. Also shown in Figure 15 are Grosch's relevant experimental results. With both "Grosch-Klüppel" and "Grosch-Fletcher" compounds, the predicted peak friction coefficients are much too high compared with Grosch but the sliding velocities at which the peaks occur match well. The question of what can be done to lower the peaks without changing the sliding velocities at which they occur naturally arises. Decreasing $q_{1}$ would do the former but it would raise the sliding velocity at which the friction peaks [1]. The remaining possibility is to 


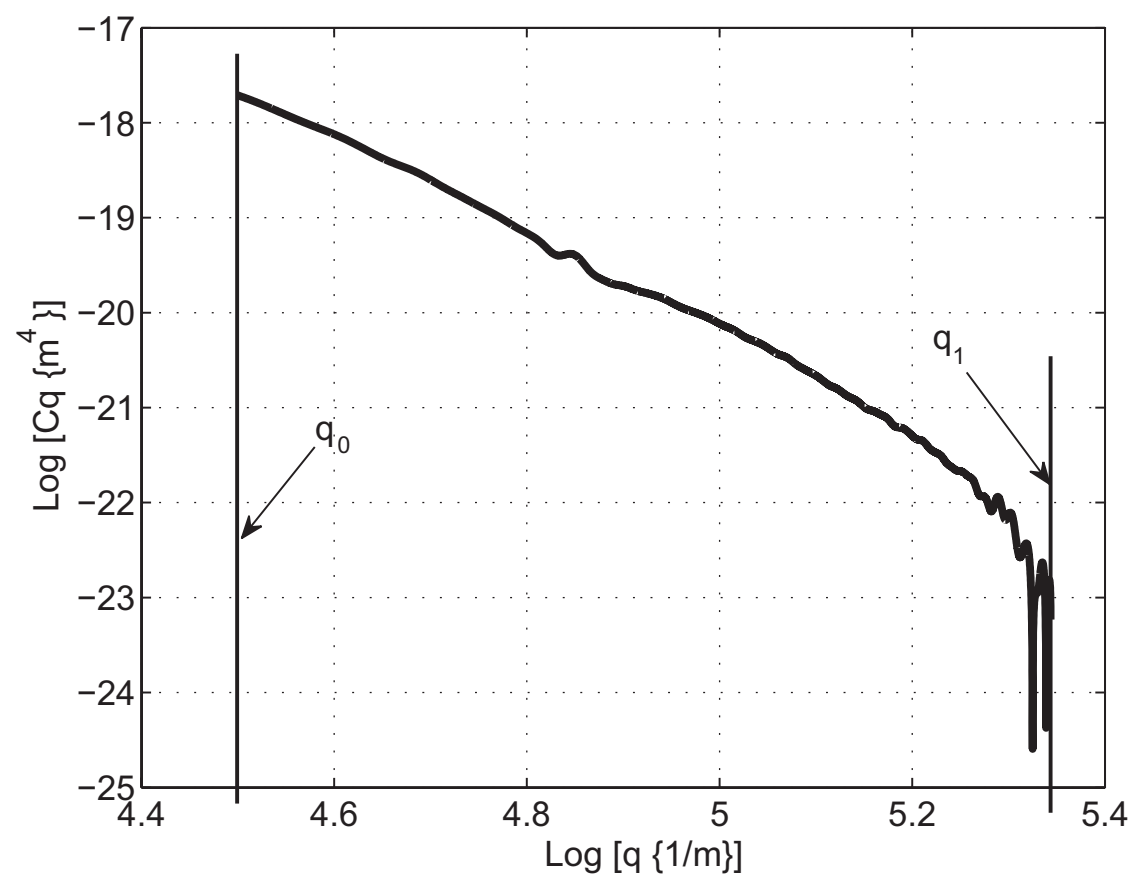

Figure 13. Displacement spectral density function of "safety walk" cloth in the wavenumber range $q_{0}$ to $q_{1}$ as measured by laser profilometer [1].

raise the storage modulus of the rubber. In order to maintain the typical characteristics of SBR (see Figure 14), this increase needs to be done without changing the shape of the modulus curve, which is illustrated in Figure 16. With this modified storage modulus, the friction coefficient results are shown in Figure 17.

Repetition of the computations in Figure 17 with the nominal contact pressure set at $1 E 5,2.5 E 5,4 E 5,5.5 E 5$ and $7 E 5 P a$ in turn showed that the real contact area fraction, $P(q)$, to be substantially proportional to the contact pressure. Consequently, the coefficient of friction was influenced only marginally, which is aligned with Grosch's observations recorded in Section 4. However, this result is at variance with the measurements shown in Figure 8.

Note that Persson [1] refers to "all the experiments known to him" giving maximal friction coefficients of order unity, contrasting with the results obtained here and with the sort of driving, braking and cornering performance observable at racing circuits.

\section{Discussion and conclusions}

Understandably, there are knowledge gaps in the record left by Grosch that can only be completely filled by repetition of the experiments conducted by him. With plausible filling of the gaps recounted here, some success in the prediction of rubber friction due to hysteresis can be achieved. The presence of a friction peak at a certain sliding speed is shown and there is sufficient freedom in the parameters to make the peak theoretical friction of a correct magnitude and to occur at a reasonable sliding speed, given the test temperature. By its nature, of course, the theory is based on viscoelasticity, so that Grosch's temperature to sliding velocity equivalence is bound to be shown.

However, the predicted friction depends too strongly on the sliding speed. For sliding speeds below the peak, it is clear that the differences between experiment and theory can 


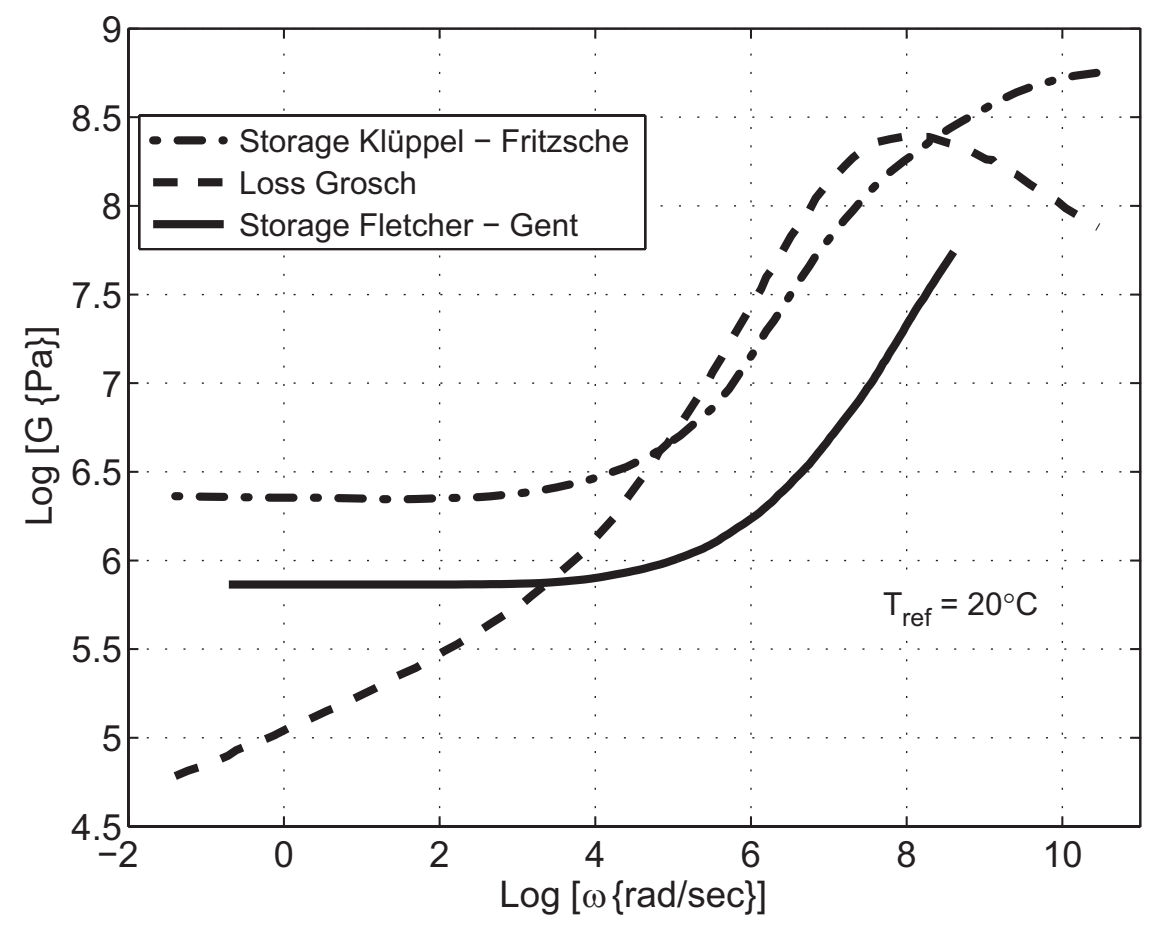

Figure 14. Storage and loss moduli curves for SBR compound. The loss modulus is as given by Grosch but the storage moduli are taken from [2] and [2], see [1].

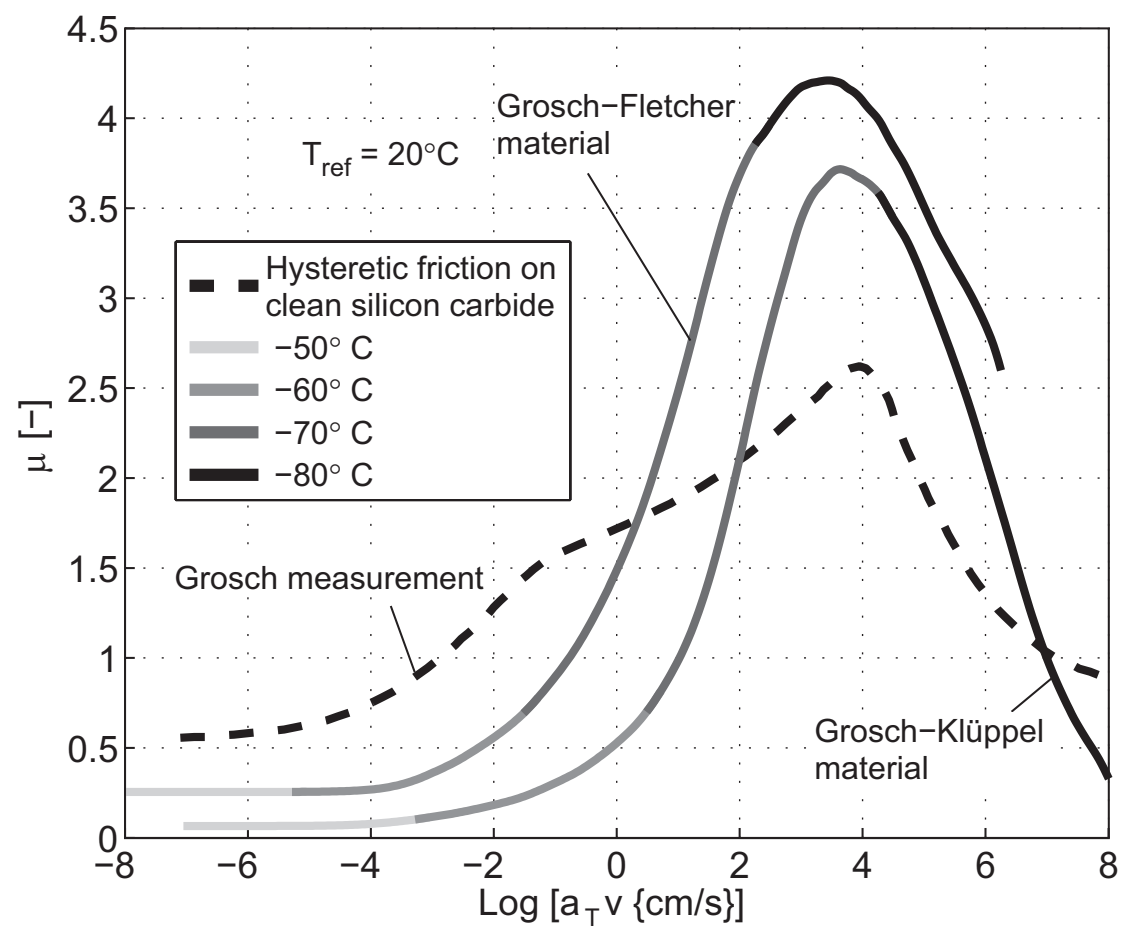

Figure 15. Simulated hysteretic friction master curves for "Grosch-Klüppel" and "Grosch-Fletcher" compounds on silicon carbide paper. The curves are compared with Grosch's measured master curve. Each segment in the simulated curves corresponds to a particular compound temperature $T$ [1]. 


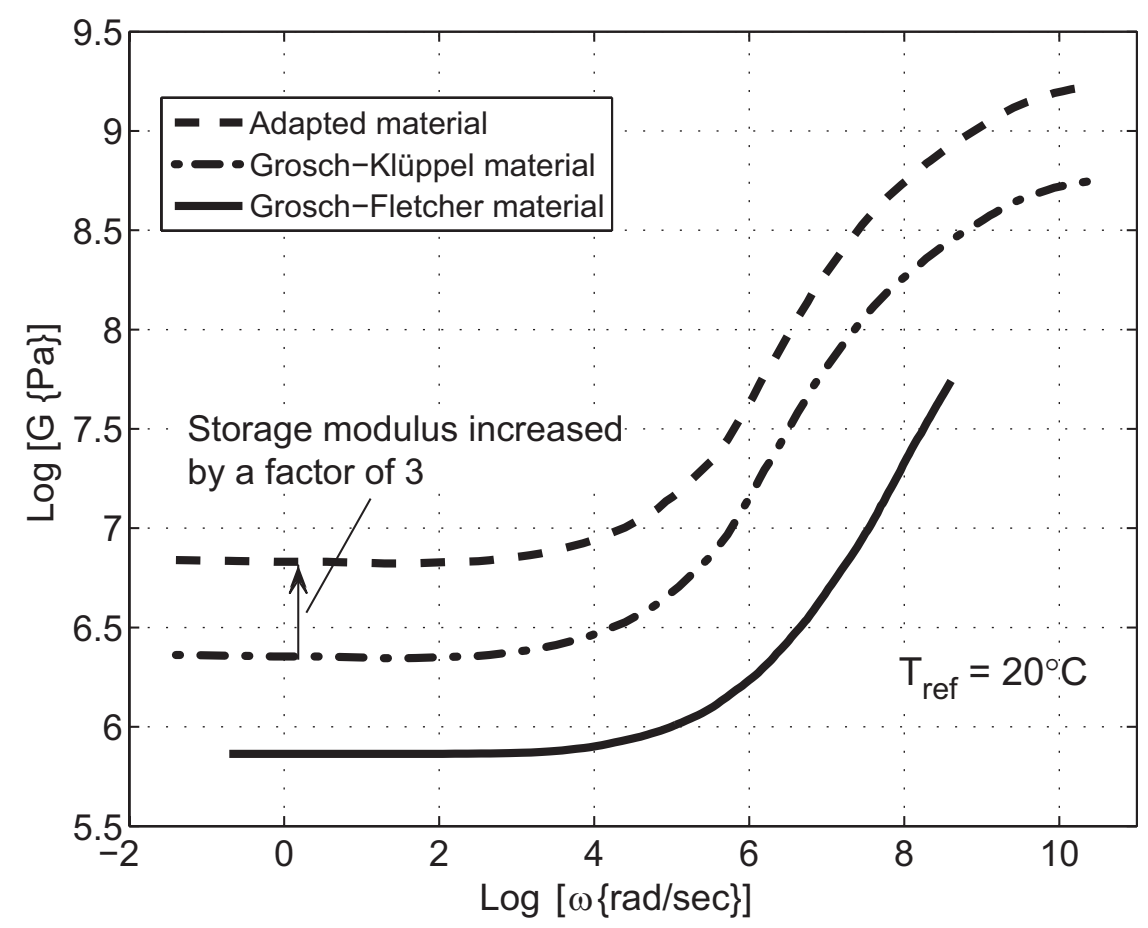

Figure 16. The assumed or "adapted" storage modulus of SBR and the storage moduli of the "Grosch-Klüppel" and the "Grosch-Fletcher" materials [1].

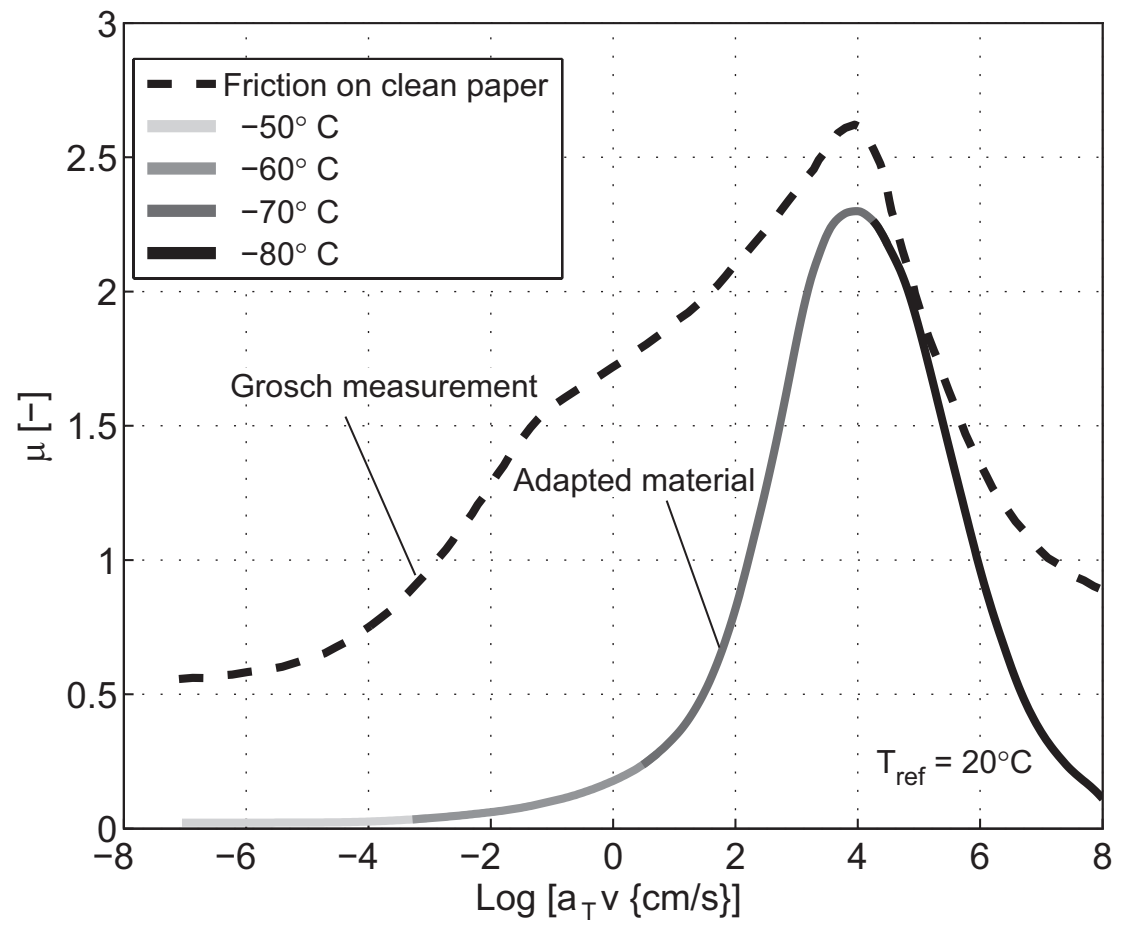

Figure 17. Grosch's friction-coefficient measurements and the simulated master curve for the "adapted material" $[1]$. 
be accounted for by the adhesion contribution to friction that is present in the experiments but not in the theory. For sliding speeds above the peak, the adhesion contribution can be expected to be small and it cannot account for the observed discrepancies. The high-sliding-speed behaviour remains problematic. Some uncertainty regarding the influence of nominal contact pressure on the friction coefficient remains. Some experiments show very little influence, others much more. Persson's theory is aligned with the former set but is at variance with the latter.

Returning to the application to tyres, roads and motor-racing mentioned at the beginning, it can be surmised that "green" tracks are mostly quite rough and that the deposition of wear debris from tyres makes them smoother. In terms of the power spectrum, the smoothened track would show an increased gradient and a smaller $q_{1}$ value compared to the "green" track. Apparently, the adhesion contribution to friction increases, if the rubber of the tyres matches the rubber laid down on the track, but the hysteretic contribution decreases as the track gets smoother. The driver searches for the peak friction and the tyre and vehicle technicians contribute to making the tyres run in the best temperature range. If rain falls on a "rubbered-in" track, the smoother racing line is affected more than the remaining surface and drivers will commonly run on that remaining surface, where maximum friction can now be obtained. The rain typically will reduce the tyre temperature, introducing another variation to the problem.

\section{References}

[1] Grosch KA. The relation between the friction and visco-elastic properties of rubber. Proceedings of the Royal Society, Series A. 1963;274(1356):21-39.

[2] Persson BNJ. Theory of rubber friction and contact mechanics. Journal of Chemical Physics. 2001; 115:3840-3860.

[3] Williams ML, Landel RF, Ferry JD. The temperature dependence of relaxation mechanisms in amorphous polymers and other glass-forming liquids. Journal of the American Chemical Society. 1955;77(14):3701-3707.

[4] Gent AN, Walter JD, editors. The pneumatic tire. National Highway Safety Administration, US Dept of Transportation; 2005. Chapter 13; p. 534-593.

[5] Grosch KA. Rubber friction and its relation to tire traction. Rubber Chemistry and Technology. 2007;80:379-411.

[6] Schallamach A. The load dependence of rubber friction. Proceedings of the Physical Society B. 1952; 65(9):657-661; doi:10.1088/0370-1301/65/9/301.

[7] Persson BNJ. Rubber friction and tire dynamics. J Phys: Condens Matter. 2011;23(1); doi:10.1088/0953-8984/23/1/015003.

[8] Lang A, Klüppel M. Modelling predictions and experimental investigations of rubber friction and tire traction. In: Gruber P, Sharp RS, editors. Proceedings of the 4th International Tyre Colloquium: Tyre models for vehicle dynamics analysis. Guildford, UK: University of Surrey; 2015. p. 228-233; isbn 978-1-84469-032-9.

[9] Lorenz B, Persson BNJ, Fortunato G, Giustiniano M, Baldoni F. Rubber friction for tire tread compound on road surfaces. Journal of Physics: Condensed Matter. 2013;25(9):095007-095014.

[10] Westermann S, Petry F, Boes R, Thielen G. Experimental investigations into the predictive capabilities of current physical rubber friction theories. Kautsch Gummi Kunstst. 2004;57:645-650.

[11] Schallamach A. A theory of dynamic rubber friction. Wear. 1963;6(5):375-382.

[12] Grosch KA. The speed and temperature dependence of rubber friction and its bearing on the skid resistance of tires. In: Hayes DL, Browne AL, editors. The physics of tire traction, theory and experiment. New York: Plenum Press; 1974. p. 143-166.

[13] Persson BNJ. Rubber friction: role of the flash temperature. J Phys: Condens Matter. 2006;18:7789 7823; doi:10.1088/0953-8984/18/32/025.

[14] Selig M, Lorenz B, Henrichmoeller D, Schmidt K, Ball A, Persson BNJ. Rubber friction and tire dynamics: A comparison of theory with experimental data. Tire Science and Technology. 2014 OctoberDecember;42(4):216-262; tSTCA. 
[15] Persson BNJ. Adhesion between an elastic body and a randomly rough hard surface. The European Physics Journal E. 2002;8:385-401; doi:10:1140/epje/12002-10025-1.

[16] Lorenz B, Persson B, Dieluweit S, Tada T. Rubber friction: Comparison of theory with experiment. The European Physical Journal E. 2011;34(12):1-11.

[17] Lorenz B, Persson BNJ. Rubber friction and tire dynamics: A comparison of theory with experimental data. In: Gruber P, Sharp RS, editors. Proceedings of the 4th International Tyre Colloquium: Tyre models for vehicle dynamics analysis. Guildford, UK: University of Surrey; 2015. p. 296-297; isbn 978-1-84469-032-9.

[18] Persson BNJ, Albohr O, Tartaglino U, Volokitin AI, Tosatti E. On the nature of surface roughness with application to contact mechanics, sealing, rubber friction and adhesion. Journal of Physics: Condensed Matter. 2005;17(1):R1-R62.

[19] Fina E, Gruber P, Sharp RS. Hysteretic rubber friction: application of persson's theories to grosch's experimental results. Trans ASME, Journal of Applied Mechanics. 2014;81(12):121001-1-121001-6; doi:10.1115/1.4028722.

[20] Klüppel M, Fritzsche J. Viscoelastic properties of filler reinforced elastomers. CRC Press; 2009.

[21] Fletcher WP, Gent AN. Dynamic shear properties of some rubber-like materials. British Journal of Applied Physics. 1957;8(5):194-201. 